Supporting Information for:

\title{
Effect of Oligomer Length on Photophysical Properties of Platinum Acetylide Donor-Acceptor-Donor Oligomers
}

\author{
Seda Cekli, ${ }^{\dagger}$ Russell W. Winkel, ${ }^{\dagger}$ Kirk S. Schanze $^{*}{ }^{\dagger}$ \\ $\dagger$ Department of Chemistry and Center for Macromolecular Science and Engineering, University \\ of Florida, Gainesville, Florida 32611-7200, United States
}

*Author to whom correspondence should be addressed; electronic mail: k.schanze@chem.ufl.edu;

Table of Content

I. Synthesis and Characterization...............................................2-11

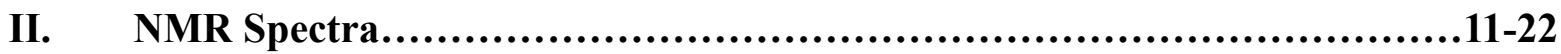

III. Electrochemical Data.......................................................23

IV. Photophysical Data.......................................................24-25

V. Computational Data.....................................................26-27

VI. References...................................................................28

Supporting Information. Cekli S., Winkel R. W., Alarousu E., Mohammed O. F., Schanze K. S. 


\section{Synthesis and Characterization}

The synthetic methodology used for the synthesis of $\mathbf{O 1}$ and $\mathbf{1}$ was explained elsewhere. ${ }^{1}$ The synthetic routes used to prepare $\mathbf{O 2}, \mathbf{O 3}$, and $\mathbf{p}$-TBT are illustrated in Scheme S1. The NMR spectra of the molecules were included in this Supporting Information. In general, acetylene containing DAD chromophores were reacted with cis- or trans-Pt complexes in the presence of a catalytic amounts of $\mathrm{CuI}$ and piperidine in toluene to afford the desired molecules.

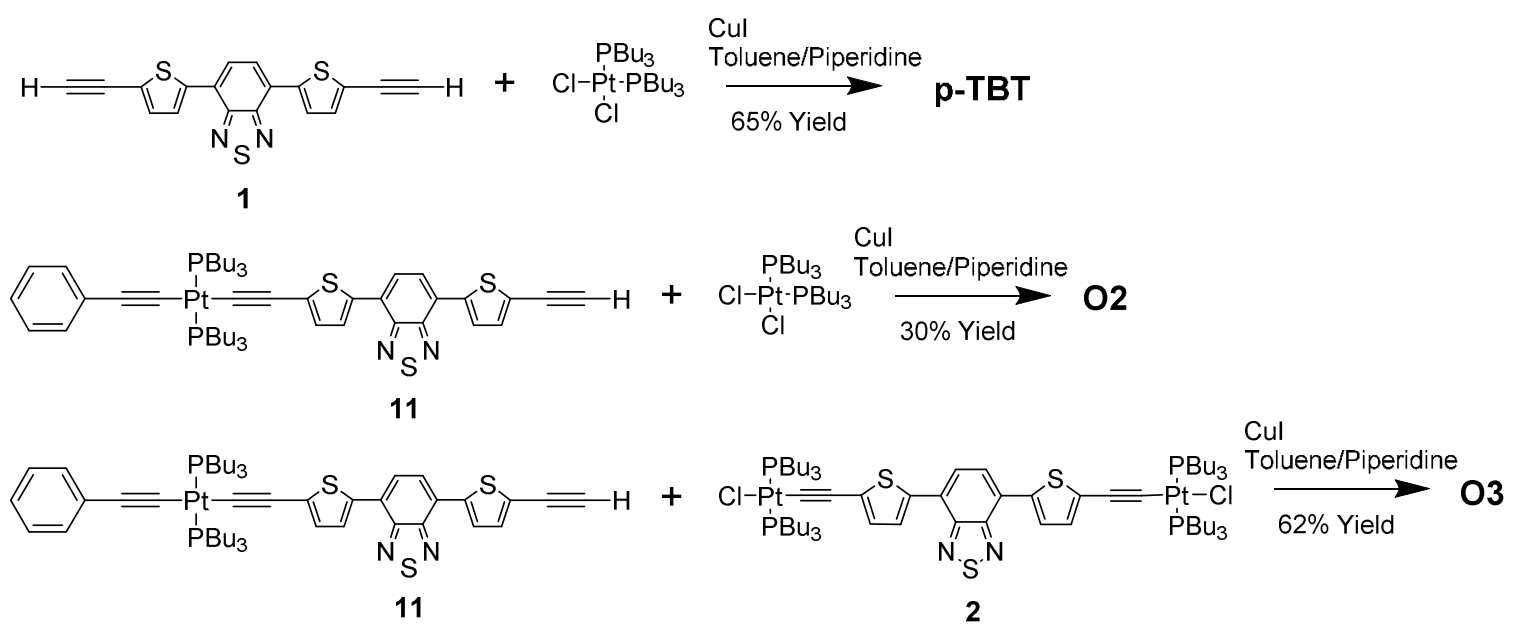

Scheme S1. Synthesis of O2, O3 and p-TBT.

Precursor $\mathbf{1 1}$ was prepared for the synthesis of $\mathbf{O 2}$ and $\mathbf{O 3}$ according to a synthetic route presented in Scheme S2. To obtain the mono brominated TBT chromophore (5), compound 3 (also referred to as TBT) was reacted with 1 equivalent of $\mathrm{N}$-bromosuccinimide in chloroform/acetic acid solution, yielding the mixture of compound 3, 4 and $\mathbf{5}$. This mixture cannot be separated by column chromatography, therefore (triisopropylsilyl)acetylene moieties were installed on the brominated sites of the chromophores by palladium-catalyzed Sonogashira reaction which is subsequently separated via column chromatography to yield desired compound 4-6 (60\% yield). Compound 7 was obtained in moderate yield upon iodination of 6 by Nbromosuccinimide in chloroform/acetic acid solution. Then, compound 7 was subjected to Stille

Supporting Information. Cekli S., Winkel R. W., Schanze K. S. 
coupling with (trimethylsilyl)acetylene in the presence of $\operatorname{Pd}(0)$ catalyst giving compound 8 . TMS group was selectively removed by $\mathrm{K}_{2} \mathrm{CO}_{3}$ at room temperature, followed by Hagihara reaction with trans-platinum complex, yielding the compound $\mathbf{1 0}$ in good yield. Finally, the TIPS group was removed by TBAF in $\mathrm{CHCl}_{3}$ to obtain the precursor 11 (80\% yield).
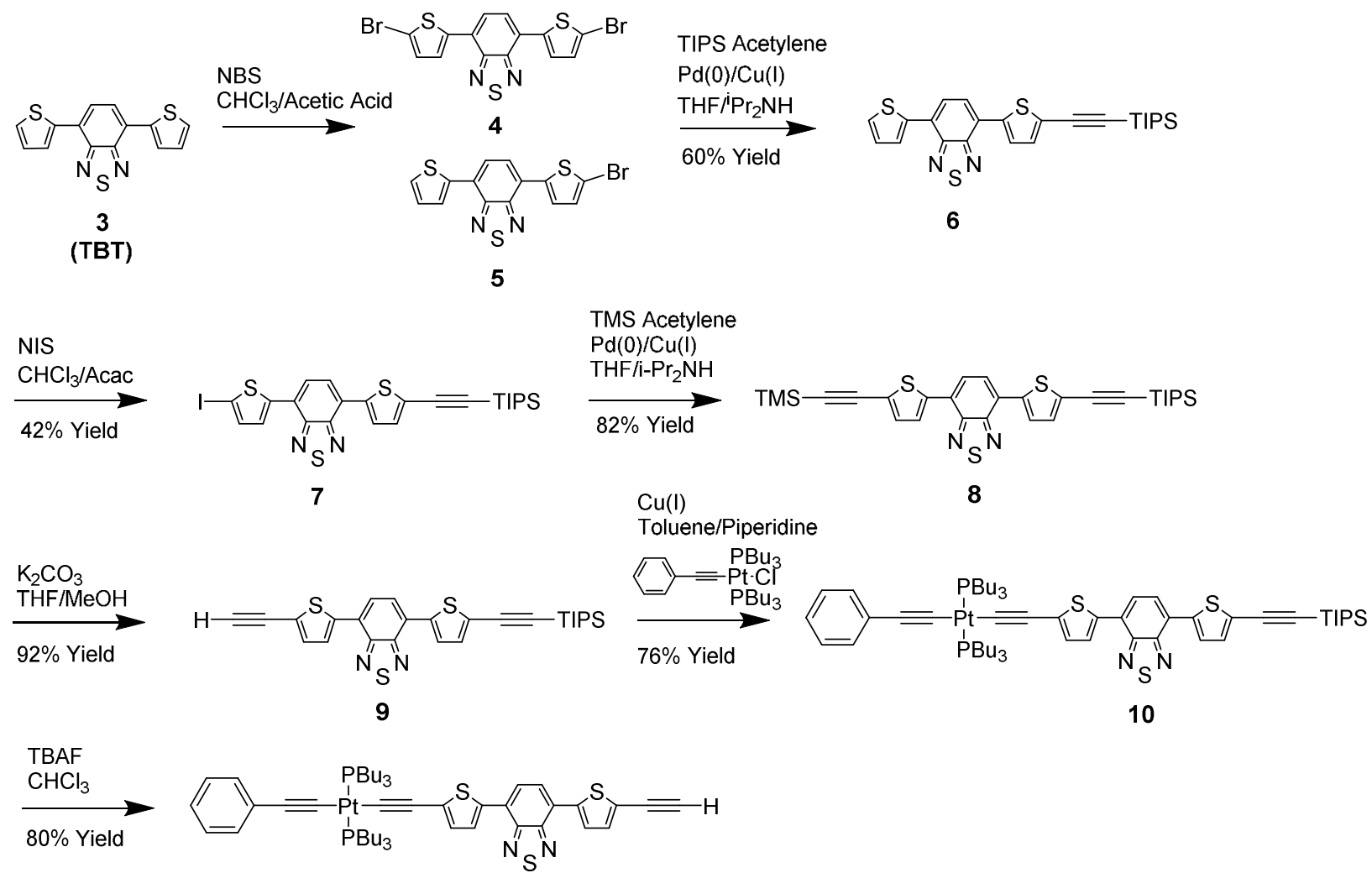

11

Scheme S2. Synthesis of Precursor 11.

Prior to the route illustrated in Scheme S1, another strategy was followed to synthesize the oligomers which involved the coupling between $\mathbf{1}$ and cis- $\mathrm{Pt}\left(\mathrm{PBu}_{3}\right)_{2} \mathrm{Cl}_{2}$ with nonstoichiometric ratios under Hagihara conditions. This strategy was adopted from Mei's Ph.D. dissertation $^{2}$ and developed to yield 2, 12 and $\mathbf{1 3}$ in a one-pot reaction (Scheme S3). In general, $\mathbf{1}$ was reacted with 3 equivalents of a cis- $\mathrm{Pt}\left(\mathrm{PBu}_{3}\right)_{2} \mathrm{Cl}_{2}$ with catalytic amounts of $\mathrm{CuCl}$ in a trimethylamine/dichloromethane mixture. It is important to note that when $\mathrm{CuI}$ is used as a catalyst, ion-exchange between iodine and chlorine takes place and oligomerization does not

Supporting Information. Cekli S., Winkel R. W., Schanze K. S. 
proceed (Figure S2 and S3). The reaction mixture was purified via column chromatography yielding to 2, 12 and $\mathbf{1 3}$ in low yields. These intermediate oligomer products were characterized by ${ }^{1} \mathrm{H}-\mathrm{NMR}$ and ${ }^{31} \mathrm{P}-\mathrm{NMR}$ (see below). In spite of a successful reaction optimization and characterization steps, we did not follow this path further to afford final compounds, because the reaction yields were too low in larger scales.
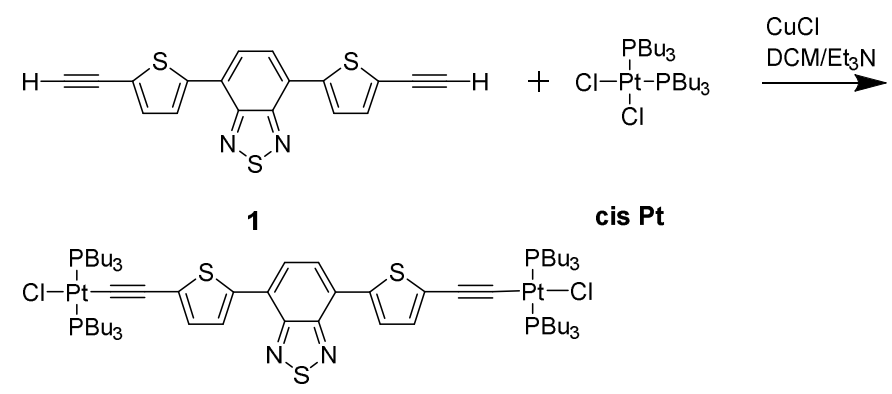

$2(22 \%$ yield $)$
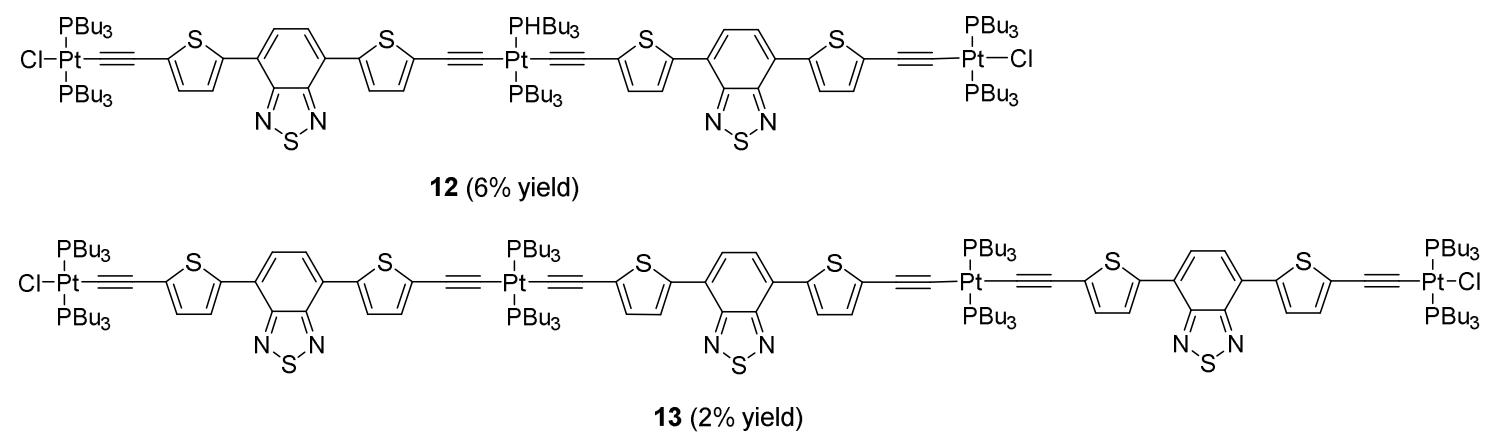

Scheme S3. One-pot synthetic route for oligomer building blocks.

In the p-TBT spectra, there are two other peaks observed at 7.14 and $0.37 \mathrm{ppm}$ which are assigned to the phosphine groups at the end of the polymer chains. In particular, the peak at 7.14 ppm corresponds to the phosphine groups on Pt-Cl (see ${ }^{31} \mathrm{P}-\mathrm{NMR}$ of 2 , Figure $\mathrm{S} 3$ ) and the one at $0.37 \mathrm{ppm}$ corresponds to the phosphine groups on Pt-I (Figure S3).

The polymer sample was also characterized by gel permeation chromatography (GPC). THF was used as the eluent, and the molecular weight was referenced to polystyrene. The GPC chromatogram of p-TBT reveals that the number-average molecular weight $(\mathrm{Mn})$ of $16.6 \mathrm{kDa}$ and a relatively narrow polydispersity index (PDI) of 2.18.

Supporting Information. Cekli S., Winkel R. W., Schanze K. S. 


\section{Materials and Instrumentation}

Starting materials used in the synthesis of oligomers and polymers, $\mathrm{Pd}\left(\mathrm{PPh}_{3}\right)_{4}, \mathrm{CuI}$ and TBAF were obtained from commercial sources. Compounds 1 to $3^{1}$ and $6^{3}$ were synthesized according to the literature procedures. All reactions were performed under argon atmosphere in anhydrous solvents, which were dried prior use by the standard procedures. Merck silica gel 60 (particle size $0.04-0.063 \mathrm{~mm}$ ) was used for flash chromatography.

\section{4-(5-iodothiophen-2-yl)-7-(5-((triisopropylsilyl)ethynyl)thiophen-2-}

yl)benzo[c] $[1,2,5]$ thiadiazole (4-7) To a solution of $0.27 \mathrm{~g}$ of 4-6 (0.72 mmol, 1 equiv) in $\mathrm{CHCl}_{3} /$ acetic acid [2:1 (v/v), $\left.60 \mathrm{~mL}\right], 0.18 \mathrm{~g}$ of NIS $(0.79 \mathrm{mmol}, 1.1$ equiv) was added. The reaction mixture was stirred at room temperature for overnight. The solvent was removed under reduced pressure. The residue was purified by silica column chromatography with $\mathrm{CH}_{2} \mathrm{Cl}_{2} /$ hexane (1:2). The desired product was obtained as orange solid $(0.18 \mathrm{~g}, 42 \%)$. ${ }^{1} \mathrm{H}-\mathrm{NMR}$ $\left(500 \mathrm{MHz}, \mathrm{CDCl}_{3}\right): 7.94(\mathrm{~d}, J=4 \mathrm{~Hz}, 1 \mathrm{H}), 7.83(\mathrm{~d}, J=7.5 \mathrm{~Hz}, 1 \mathrm{H}), 7.80(\mathrm{~d}, J=7.5 \mathrm{~Hz}, 1 \mathrm{H})$, $7.70(\mathrm{~d}, J=4 \mathrm{~Hz}, 1 \mathrm{H}), 7.35(\mathrm{~d}, J=4 \mathrm{~Hz}, 1 \mathrm{H}), 7.30(\mathrm{~d}, J=4 \mathrm{~Hz}, 1 \mathrm{H}), 1.15(\mathrm{~d}, J=2.5 \mathrm{~Hz}, 21 \mathrm{H})$; ${ }^{13} \mathrm{C}-\mathrm{NMR}\left(75.4 \mathrm{MHz}, \mathrm{CDCl}_{3}\right): 152.16,152.07,145.01,140.03,137.77,133.28,128.39,127.11$, $125.45,125.38,125.19,125.05,124.99,99.60,97.39,75.65,18.86,11.49 ;$ HRMS (ESI) Calculated for $\mathrm{C}_{25} \mathrm{H}_{27} \mathrm{IN}_{2} \mathrm{~S}_{3} \mathrm{Si}(\mathrm{M}+\mathrm{H})^{+}: m / z$ 606.0176. Found: $m / z$ 606.0145.

\section{4-(5-((triisopropylsilyl)ethynyl)thiophen-2-yl)-7-(5-((trimethylsilyl)ethynyl)thiophen-}

2-yl)benzo[c][1,2,5]thiadiazole (4-8) A solution of $0.18 \mathrm{~g}$ of 4-7 (0.3 mmol, 1 equiv) and $35 \mathrm{mg}$ of TMS acetylene (0.36 mmol, 1.2 equiv) in THF/isopropyl amine [3:2 (v/v), $50 \mathrm{~mL}$ ] was degassed for $45 \mathrm{~min}$ by bubbling with argon. $10 \mathrm{mg}$ of $\mathrm{Pd}\left(\mathrm{PPh}_{3}\right)_{4}(0.09 \mathrm{mmol}, 0.03$ equiv) was added under argon and the resulting mixture was stirred at $80{ }^{\circ} \mathrm{C}$ for 2 hours. The solvent was removed under reduced pressure. The residue was washed with water, extracted with $\mathrm{CH}_{2} \mathrm{Cl}_{2}$,

Supporting Information. Cekli S., Winkel R. W., Schanze K. S. 
and dried over $\mathrm{MgSO}_{4}$. The residue was purified by silica column chromatography with ethyl acetate/hexane (1/5). The desired product was obtained as red-orange solid $(0.14 \mathrm{~g}, 82 \%) .{ }^{1} \mathrm{H}-$ NMR (500 MHz, $\left.\mathrm{CDCl}_{3}\right): 7.94$ (dd, $\left.J=5.4,3.9 \mathrm{~Hz}, 2 \mathrm{H}\right), 7.83$ (s, 2H), 7.30 (dd, $J=3.9,2.1 \mathrm{~Hz}$, 2H), $1.15(\mathrm{~d}, J=2.5 \mathrm{~Hz}, 21 \mathrm{H}), 0.28(\mathrm{~s}, 9 \mathrm{H}),{ }^{13} \mathrm{C}-\mathrm{NMR}\left(75.4 \mathrm{MHz}, \mathrm{CDCl}_{3}\right)$ : 152.16, 140.37, $140.02,133.41,133.26,127.07,127.06,125.44,125.38,123.32,125.29,125.19,124.69,101.17$, 99.62, 97.99, 97.80, 18.85, 11.49, 0.04; HRMS (ESI) Calculated for $\mathrm{C}_{30} \mathrm{H}_{36} \mathrm{~N}_{2} \mathrm{~S}_{3} \mathrm{Si}_{2}(\mathrm{M}+\mathrm{H})^{+}: \mathrm{m} / z$ 576.1573. Found: $m / z 576.1582$.

\section{4-(5-ethynylthiophen-2-yl)-7-(5-((triisopropylsilyl)ethynyl)thiophen-2-}

yl)benzo[c][1,2,5]thiadiazole (4-9). A solution of $0.14 \mathrm{~g}$ of $4-8(0.24 \mathrm{mmol}, 1$ equiv) and $68 \mathrm{mg}$ of $\mathrm{K}_{2} \mathrm{CO}_{3}(0.49 \mathrm{mmol}, 2$ equiv) in THF/methanol $[1: 1(\mathrm{v} / \mathrm{v}), 40 \mathrm{~mL}]$ was stirred at room temperature overnight. The solvent was removed under reduced pressure. The residue was washed with water, extracted with $\mathrm{CH}_{2} \mathrm{Cl}_{2}$, and dried over $\mathrm{MgSO}_{4}$. The residue was purified by silica column chromatography with dichloromethane/hexane (1/2). The desired product was obtained as red-orange solid (0.11 g, 92\%). ${ }^{1} \mathrm{H}-\mathrm{NMR}\left(500 \mathrm{MHz}, \mathrm{CDCl}_{3}\right)$ : 7.95 (ddd, $J=8.1,3.9$, $1.5 \mathrm{~Hz}, 2 \mathrm{H}), 7.84(\mathrm{~d}, J=2.8 \mathrm{~Hz}, 2 \mathrm{H}), 7.35(\mathrm{~d}, J=3.9 \mathrm{~Hz}, 2 \mathrm{H}), 7.30(\mathrm{~d}, J=3.9 \mathrm{~Hz}, 2 \mathrm{H}), 3.48(\mathrm{~s}$, $1 \mathrm{H}), 1.15(\mathrm{~d}, J=2.6 \mathrm{~Hz}, 21 \mathrm{H}) ;{ }^{13} \mathrm{C}-\mathrm{NMR}\left(75.4 \mathrm{MHz}, \mathrm{CDCl}_{3}\right): 152.22,140.71,139.99,133.92$, $133.28,127.16,127.05,125.67,125.60,125.35,125.30,125.21,123.38,99.58,98.04,83.19$, 77.20, 18.84, 11.48; HRMS (ESI) Calculated for $\mathrm{C}_{27} \mathrm{H}_{28} \mathrm{~N}_{2} \mathrm{~S}_{3} \mathrm{Si}(\mathrm{M}+\mathrm{H})^{+}: m / z$ 504.1178. Found: $\mathrm{m} / \mathrm{z} 504.1163$.

4-10. A solution of $0.11 \mathrm{~g}$ of $4-9(0.22 \mathrm{mmol}, 1$ equiv $)$ and $0.18 \mathrm{~g}$ of transethynylphenylchlorobis(tri-n-butylphosphine)platinum(II) $\quad\left(\begin{array}{lllll}0.25 & \mathrm{mmol}, & 1.1 & \text { equiv }) & \text { in }\end{array}\right.$ toluene/piperidine [1:1 (v/v), $40 \mathrm{~mL}]$ was degassed for $45 \mathrm{~min}$ by bubbling with argon and was stirred at room temperature overnight, in the presence of catalytic amount of $\mathrm{CuI}(2 \mathrm{mg}, 0.05$

Supporting Information. Cekli S., Winkel R. W., Schanze K. S. 
equiv). The solvent was removed under reduced pressure. The residue was washed with water, extracted with $\mathrm{CH}_{2} \mathrm{Cl}_{2}$, and dried over $\mathrm{MgSO}_{4}$. The residue was purified by flash silica column chromatography with dichloromethane/hexane (2/8). The desired product was obtained as dark red-purple viscous liquid $(0.20 \mathrm{~g}, 76 \%) .{ }^{1} \mathrm{H}-\mathrm{NMR}\left(500 \mathrm{MHz}, \mathrm{CD}_{2} \mathrm{Cl}_{2}\right): 8.02(\mathrm{~d}, J=3.8 \mathrm{~Hz}, 1 \mathrm{H})$, $7.94(\mathrm{~d}, J=4.2 \mathrm{~Hz}, 1 \mathrm{H}), 7.86(\mathrm{~d}, J=8.1 \mathrm{~Hz}, 1 \mathrm{H}), 7.78(\mathrm{~d}, J=7.8 \mathrm{~Hz}, 1 \mathrm{H}), 7.31(\mathrm{~d}, J=3.9 \mathrm{~Hz}$, 1H), $7.28-7.16(\mathrm{~m}, 3 \mathrm{H}), 7.11(\mathrm{t}, \mathrm{J}=7.3 \mathrm{~Hz}, 1 \mathrm{H}), 6.92(\mathrm{~d}, \mathrm{~J}=3.7 \mathrm{~Hz}, 1 \mathrm{H}), 2.17-2.14(\mathrm{~m}, 12 \mathrm{H})$, $1.64-1.62(\mathrm{~m}, 12 \mathrm{H}), 1.50-1.43(\mathrm{~m}, 12 \mathrm{H}), 1.16(\mathrm{~s}, 21 \mathrm{H}), 0.97-0.91(\mathrm{~m}, 18 \mathrm{H}) ;{ }^{13} \mathrm{C}-\mathrm{NMR}(75.4$ $\left.\mathrm{MHz}, \mathrm{CDCl}_{3}\right): 152.70,152.62,140.68,135.73,133.31,132.17,130.90,19.06,128.63,128.28$, $127.99,127.95,126.84,126.56,126.08,125.04,124.88,124.66,124.57,124.31,109.50,109.00$, 107.44, 101.82, 99.74, 97.61, 26.53, 24.56, 24.13, 18.83, 14.00, 11.49; ${ }^{31} \mathrm{P}-\mathrm{NMR}(121.44 \mathrm{MHz}$, $\left.\mathrm{CDCl}_{3}\right): 3.29\left(J_{\mathrm{Pt}-\mathrm{P}}=2340 \mathrm{~Hz}\right)$; HRMS (ESI) Calculated for $\mathrm{C}_{59} \mathrm{H}_{86} \mathrm{~N}_{2} \mathrm{P}_{2} \mathrm{PtS}_{3} \mathrm{Si}(\mathrm{M}+\mathrm{H})^{+}: \quad m / z$ 1204.4851. Found: $m / z$ 1204.4720.

4-11. A solution of $30 \mathrm{mg}$ of $4-10$ (0.025 mmol, 1 equiv) and $9.8 \mathrm{mg}$ TBAF (0.0374 mmol, 1.5 equiv) in chloroform was degassed for $45 \mathrm{~min}$ by bubbling with argon and was stirred at room temperature overnight for 2 hours. The solvent was removed under reduced pressure. The residue was washed with water, extracted with $\mathrm{CH}_{2} \mathrm{Cl}_{2}$, and dried over $\mathrm{MgSO}_{4}$. The residue was passed through silica by dichloromethane. The desired product was obtained as dark redpurple solid (21 mg, 80\%). ${ }^{1} \mathrm{H}-\mathrm{NMR}\left(500 \mathrm{MHz}, \mathrm{CD}_{2} \mathrm{Cl}_{2}\right): 8.02$ (d, $\left.J=3.8 \mathrm{~Hz}, 1 \mathrm{H}\right), 7.94$ (d, $J=$ $4.2 \mathrm{~Hz}, 1 \mathrm{H}), 7.86(\mathrm{~d}, J=8.1 \mathrm{~Hz}, 1 \mathrm{H}), 7.78(\mathrm{~d}, J=7.8 \mathrm{~Hz}, 1 \mathrm{H}), 7.31$ (d, $J=3.9 \mathrm{~Hz}, 1 \mathrm{H}), 7.28-$ $7.16(\mathrm{~m}, 3 \mathrm{H}), 7.11(\mathrm{t}, \mathrm{J}=7.3 \mathrm{~Hz}, 1 \mathrm{H}), 6.92(\mathrm{~d}, \mathrm{~J}=3.7 \mathrm{~Hz}, 1 \mathrm{H}), 3.55(\mathrm{~s}, 1 \mathrm{H}), 2.17-2.14(\mathrm{~m}$, 12H), $1.64-1.62(\mathrm{~m}, 12 \mathrm{H}), 1.50-1.43(\mathrm{~m}, 12 \mathrm{H}), 0.97-0.91(\mathrm{~m}, 18 \mathrm{H}) ;{ }^{13} \mathrm{C}-\mathrm{NMR}(75.4 \mathrm{MHz}$, $\left.\mathrm{CDCl}_{3}\right): 152.44,152.37,141.18,135.56,133.84,132.16,130.80,128.96,128.53,128.26$, $127.91,127.86,126.82,126.45,126.04,124.97,124.81,124.29,123.85,122.72,119.41,109.44$

Supporting Information. Cekli S., Winkel R. W., Schanze K. S. 
107.34, 101.78, 82.89, 77.27, 26.45, 24.48, 24.05, 13.93.; ${ }^{31} \mathrm{P}-\mathrm{NMR}\left(121.44 \mathrm{MHz}, \mathrm{CDCl}_{3}\right): 3.29$ $\left(J_{\mathrm{Pt}-\mathrm{P}}=2340 \mathrm{~Hz}\right)$; HRMS (ESI) Calculated for $\mathrm{C}_{50} \mathrm{H}_{66} \mathrm{~N}_{2} \mathrm{P}_{2} \mathrm{PtSi}(\mathrm{M}+\mathrm{H})^{+}: m / z$ 1048.3517. Found: $m / z 1048.3380$.

O2. A solution of $17 \mathrm{mg}$ of $4-11(0.025 \mathrm{mmol}, 1$ equiv) and $59 \mathrm{mg}$ of cisplatinum complex (0.056 mmol, 2.2 equiv) in toluene/piperidine [1:1 (v/v), $30 \mathrm{~mL}]$ was degassed for 45 min by bubbling with argon and was stirred at room temperature for 4 hours, in the presence of catalytic amount of $\mathrm{CuI}(1 \mathrm{mg}, 0.05$ equiv). The solvent was removed under reduced pressure. The residue was washed with water, extracted with $\mathrm{CH}_{2} \mathrm{Cl}_{2}$, and dried over $\mathrm{MgSO}_{4}$. The residue was purified by silica column chromatography with dichloromethane/hexane (1/1). The desired product was obtained as purple viscous liquid $(0.20 \mathrm{mg}, 30 \%)$. ${ }^{1} \mathrm{H}-\mathrm{NMR}\left(500 \mathrm{MHz}, \mathrm{CD}_{2} \mathrm{Cl}_{2}\right)$ : $7.99(\mathrm{~d}, \mathrm{~J}=3.5 \mathrm{~Hz}, 4 \mathrm{H}), 7.76(\mathrm{~s}, 4 \mathrm{H}), 7.27-7.18(\mathrm{~m}, 8 \mathrm{H}), 7.12(\mathrm{t}, \mathrm{J}=7.3 \mathrm{~Hz}, 2 \mathrm{H}), 6.92(\mathrm{dd}, \mathrm{J}=$ 6.3, 3.9 Hz, 4H), $2.17-2.14(\mathrm{~m}, 36 \mathrm{H}), 1.64-1.62(\mathrm{~m}, 36 \mathrm{H}), 1.50-1.43(\mathrm{~m}, 36 \mathrm{H}), 0.97-0.91$ (m, 54H); ${ }^{13} \mathrm{C}-\mathrm{NMR}\left(75.4 \mathrm{MHz}, \mathrm{CDCl}_{3}\right): 152.87,136.36,136.17,131.54,131.29,130.92$, $129.11,128.69,128.58,128.00,127.70,127.64,125.61,125.48,125.11,125.03,109.46,107.56$, 102.24, 101.83, 26.57, 26.55, 24.57, 24.13, 14.05, 14.02.; ${ }^{31}$ P-NMR (121.44 MHz, $\left.\mathrm{CDCl}_{3}\right): 3.47$ $\left(J_{\mathrm{Pt}-\mathrm{P}}=2325 \mathrm{~Hz}\right), 3.27(J$ Pt-P $=2342 \mathrm{~Hz})$; HRMS (ESI) Calculated for $\mathrm{C}_{124} \mathrm{H}_{184} \mathrm{~N}_{4} \mathrm{P}_{6} \mathrm{Pt}_{3} \mathrm{~S}_{6}$ $(\mathrm{M}+\mathrm{H})^{+}: m / z$ 6629.1513. Found: $m / z 6629.1580$.

O3. A solution of $48 \mathrm{mg}$ of 4-11 (0.03 mmol, 1 equiv) and $38 \mathrm{mg}$ of $4-2(0.036 \mathrm{mmol}$, 1.2 equiv) in toluene/piperidine [1:1 (v/v), $30 \mathrm{~mL}]$ was degassed for 45 min by bubbling with argon and was stirred at room temperature for 4 hours, in the presence of catalytic amount of $\mathrm{CuI}$ ( $1 \mathrm{mg}, 0.05$ equiv). The solvent was removed under reduced pressure. The residue was washed with water, extracted with $\mathrm{CH}_{2} \mathrm{Cl}_{2}$, and dried over $\mathrm{MgSO}_{4}$. The residue was purified by silica column chromatography with dichloromethane/hexane (1/1). The desired product was obtained 
as purple viscous liquid $(0.27 \mathrm{mg}, 62 \%) .{ }^{1} \mathrm{H}-\mathrm{NMR}\left(500 \mathrm{MHz}, \mathrm{CD}_{2} \mathrm{Cl}_{2}\right): 8.01-7.98(\mathrm{~m}, 2 \mathrm{H})$, $7.77(\mathrm{~d}, \mathrm{~J}=3.2 \mathrm{~Hz}, 3 \mathrm{H}), 7.27-7.24(\mathrm{~m}, 1 \mathrm{H}), 7.20$ (t, J = 7.6 Hz, 1H), $7.14-7.09$ (m, 0H), 6.92 $(\mathrm{dd}, \mathrm{J}=7.4,4.0 \mathrm{~Hz}, 3 \mathrm{H}), 2.25-2.05(\mathrm{~m}, 7 \mathrm{H}), 1.70-1.59(\mathrm{~m}, 9 \mathrm{H}), 1.59-1.44(\mathrm{~m}, 17 \mathrm{H}), 1.03-$ 0.91 (m, 29H). ${ }^{13} \mathrm{C}-\mathrm{NMR}\left(75.4 \mathrm{MHz}, \mathrm{CDCl}_{3}\right): 152.86,136.36,136.34,136.17,131.53,131.33$, $131.28,130.91,129.10,128.69,128.59,128.00,127.70,127.67,127.64,125.61,125.55,125.48$ 125.10, 26.54, 24.57, 24.13, 14.05. ${ }^{31} \mathrm{P}-\mathrm{NMR}\left(121.44 \mathrm{MHz}, \mathrm{CDCl}_{3}\right): 3.47\left(J_{\mathrm{Pt}-\mathrm{P}}=2325 \mathrm{~Hz}\right), 3.27$ $(J$ Pt-P $=2342 \mathrm{~Hz})$; HRMS (ESI) Calculated for $\mathrm{C}_{166} \mathrm{H}_{244} \mathrm{~N}_{6} \mathrm{P}_{8} \mathrm{Pt}_{4} \mathrm{~S}_{9}(\mathrm{M}+\mathrm{H})^{+}: \quad m / z 3639.3264$. Found: $m / z 3639.5951$.

p-TBT. $70 \mathrm{mg}$ of $4-1(0.20 \mathrm{mmol}, 1$ equiv) and $135 \mathrm{mg}$ of cis-platinum complex $(0.20$ mmol, 1 equiv) solution in toluene/piperidine $[1: 1(\mathrm{v} / \mathrm{v}), 40 \mathrm{~mL}]$ was degassed for $45 \mathrm{~min}$ by bubbling with argon. Then catalytic amount of $\mathrm{CuI}(1.0 \mathrm{mg}$. 0.05 equiv) was added to reaction mixture. The mixture was stirred at room temperature for 2 days and then passed through a bed of silica to remove catalysts. The solvent was removed under reduced pressure. The dark-purple viscous liquid further purified by Soxhlet extraction by methanol, hexane, and THF. The desired polymer was obtained as purple viscous liquid. ${ }^{1} \mathrm{H}-\mathrm{NMR}$ (500 MHz, $\mathrm{CDCl}_{3}$ ): 7.99 (brs, 2H), 7.73 (brs, 2H), 6.93 (brs, 2H), 2.18 (brs, 12H), $1.70-1.42$ (m, 24H), 0.98 (brs, 18H); ${ }^{31} \mathrm{P}-\mathrm{NMR}$ $\left(121.44 \mathrm{MHz}, \mathrm{CDCl}_{3}\right): 7.14,3.46\left(J_{\mathrm{Pt}-\mathrm{P}}=2325 \mathrm{~Hz}\right), 0.37$; GPC: $\mathrm{M}_{\mathrm{n}}=16,600 \mathrm{~g} / \mathrm{mol}, \mathrm{PDI}=2.18$.

Supporting Information. Cekli S., Winkel R. W., Schanze K. S. 


\section{NMR Spectra}

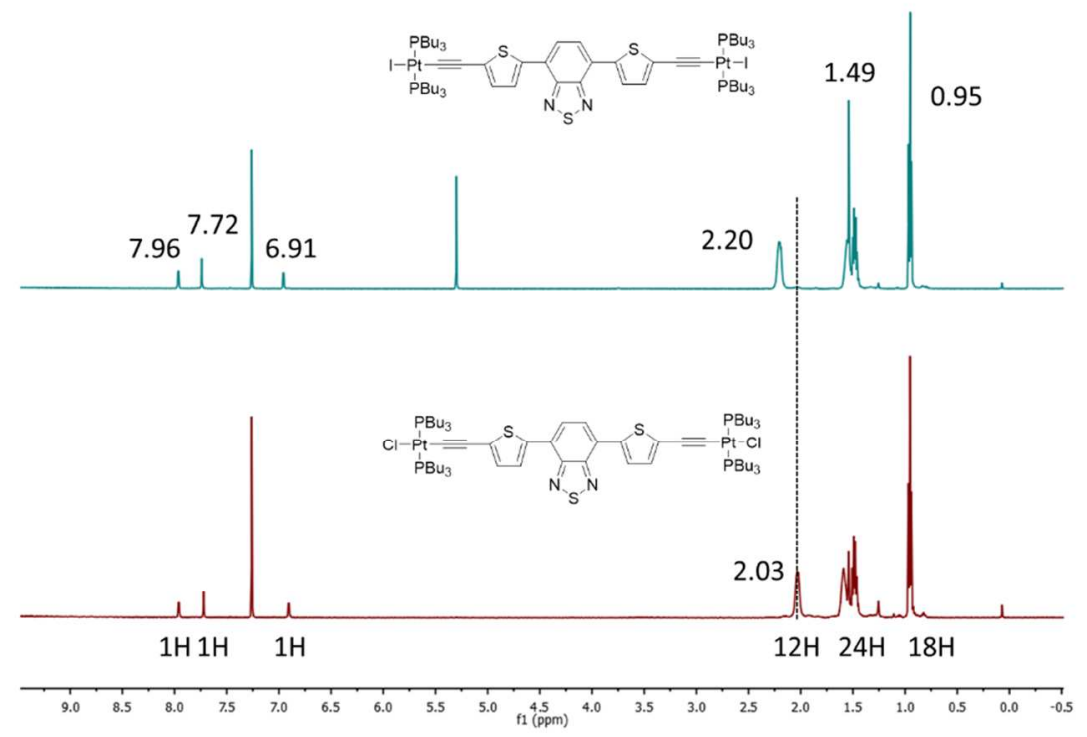

Figure S1. H-NMR of iodinated oligomerization product and 2.
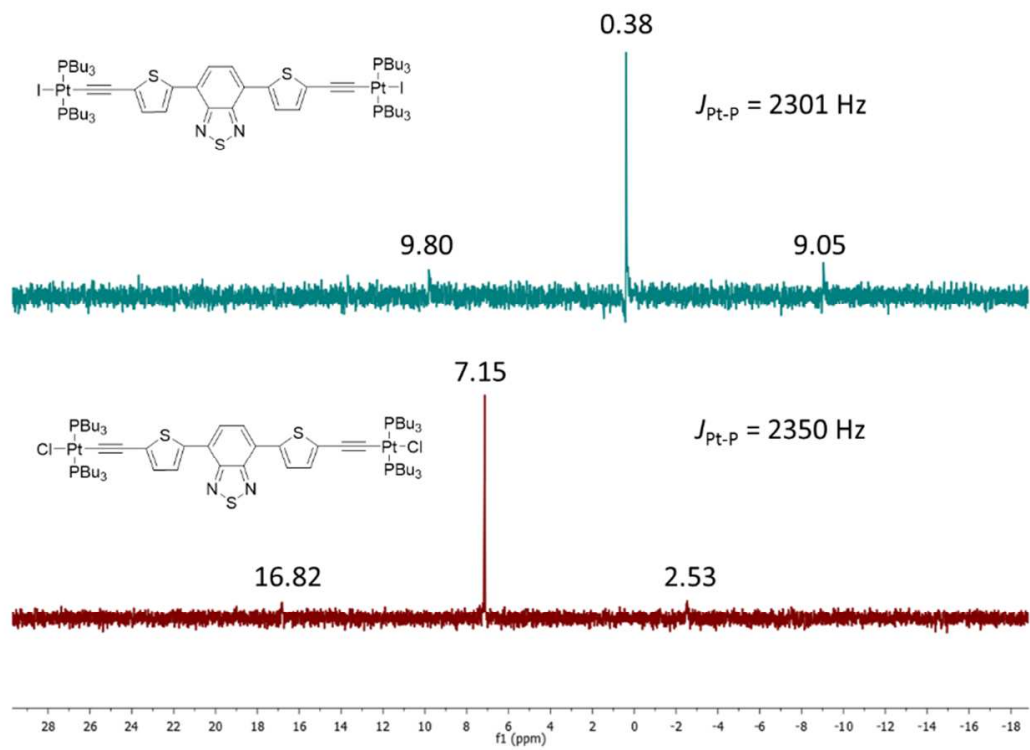

Figure S2. P-NMR of iodinated oligomerization product and 2.

Supporting Information. Cekli S., Winkel R. W., Schanze K. S. 

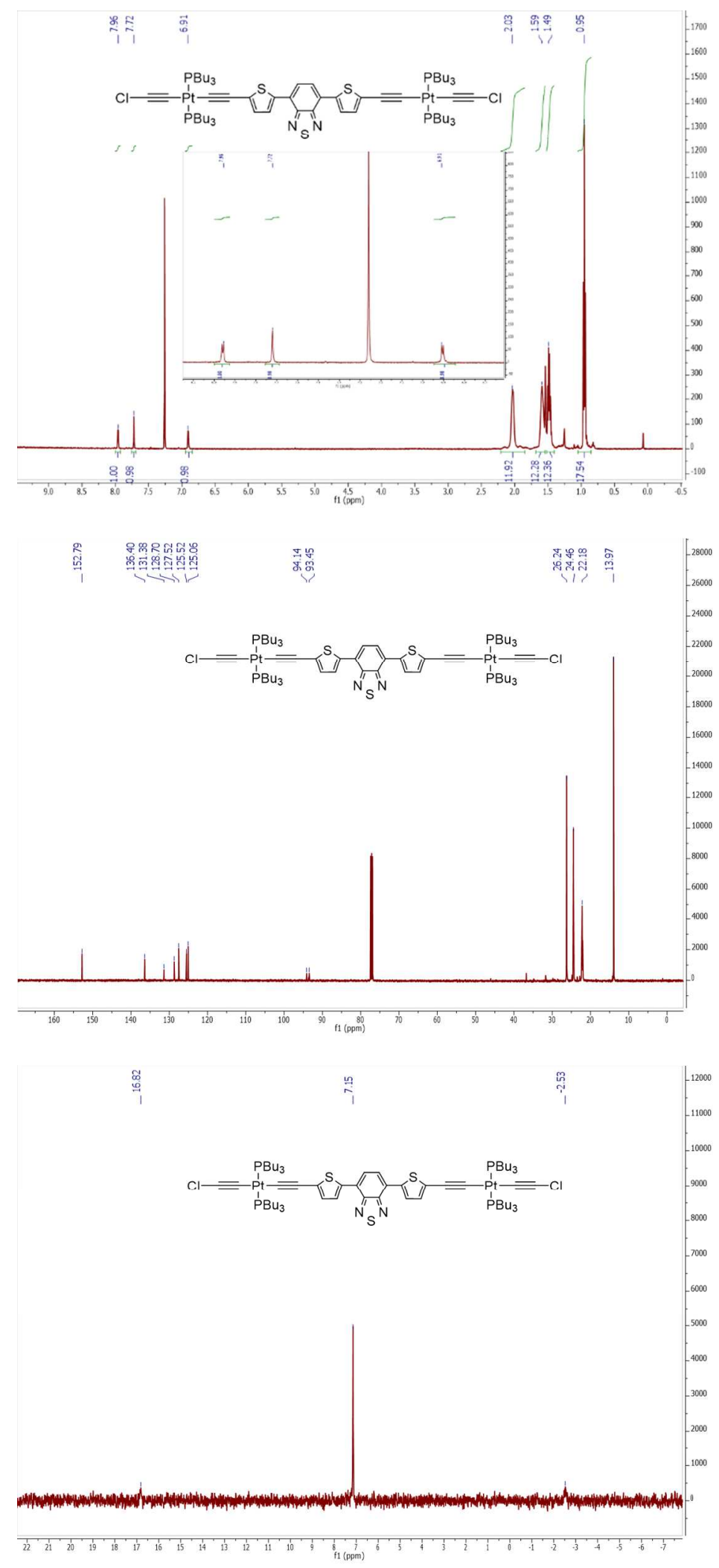

Figure S3. H and P-NMR of 2.

Supporting Information. Cekli S., Winkel R. W., Schanze K. S. 

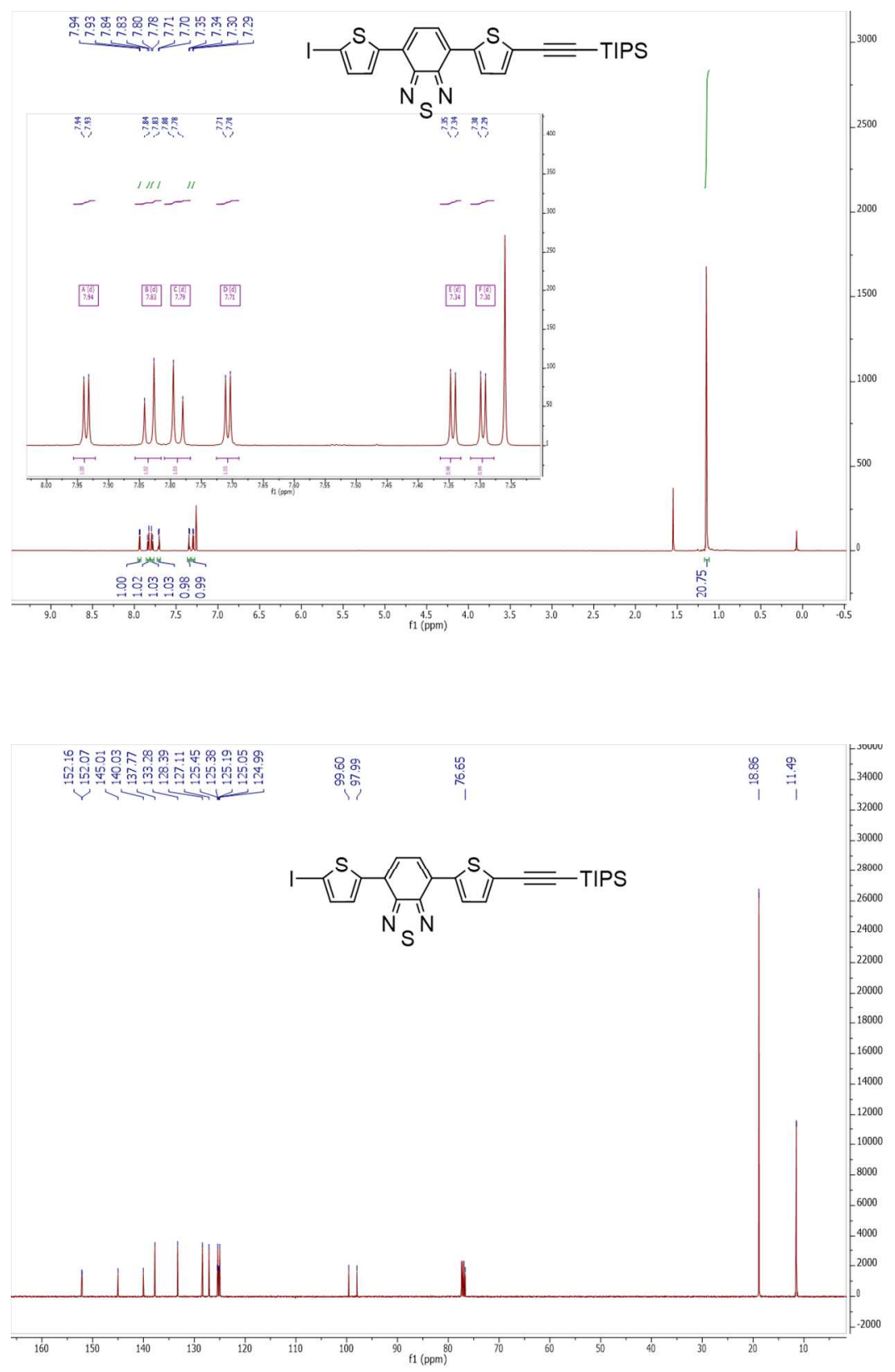

Figure S4. H and C-NMR of 7.

Supporting Information. Cekli S., Winkel R. W., Schanze K. S. 

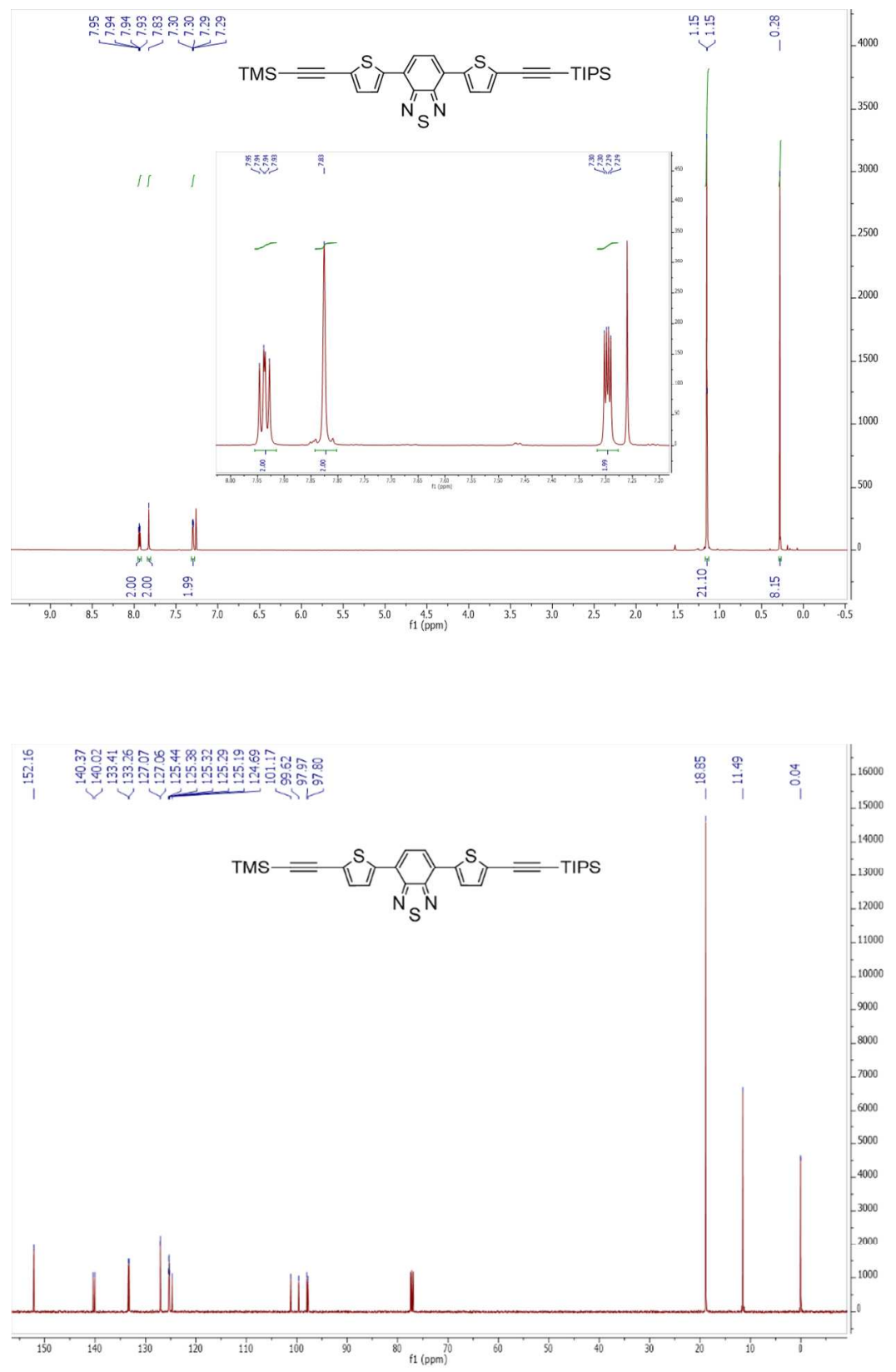

Figure S5. H and C-NMR of 8.

Supporting Information. Cekli S., Winkel R. W., Schanze K. S. 

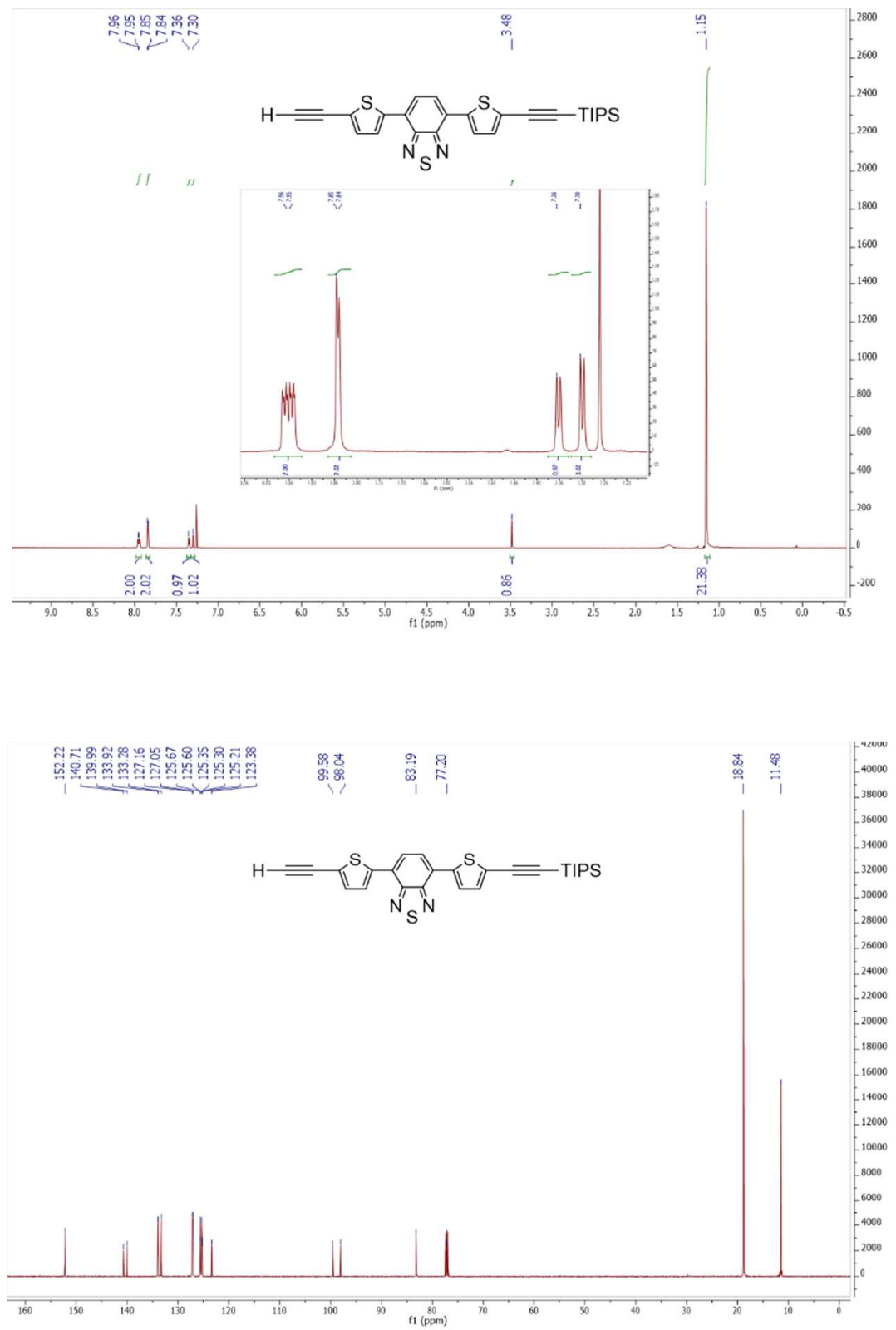

Figure S6. H and C-NMR of 9.

Supporting Information. Cekli S., Winkel R. W., Schanze K. S. 

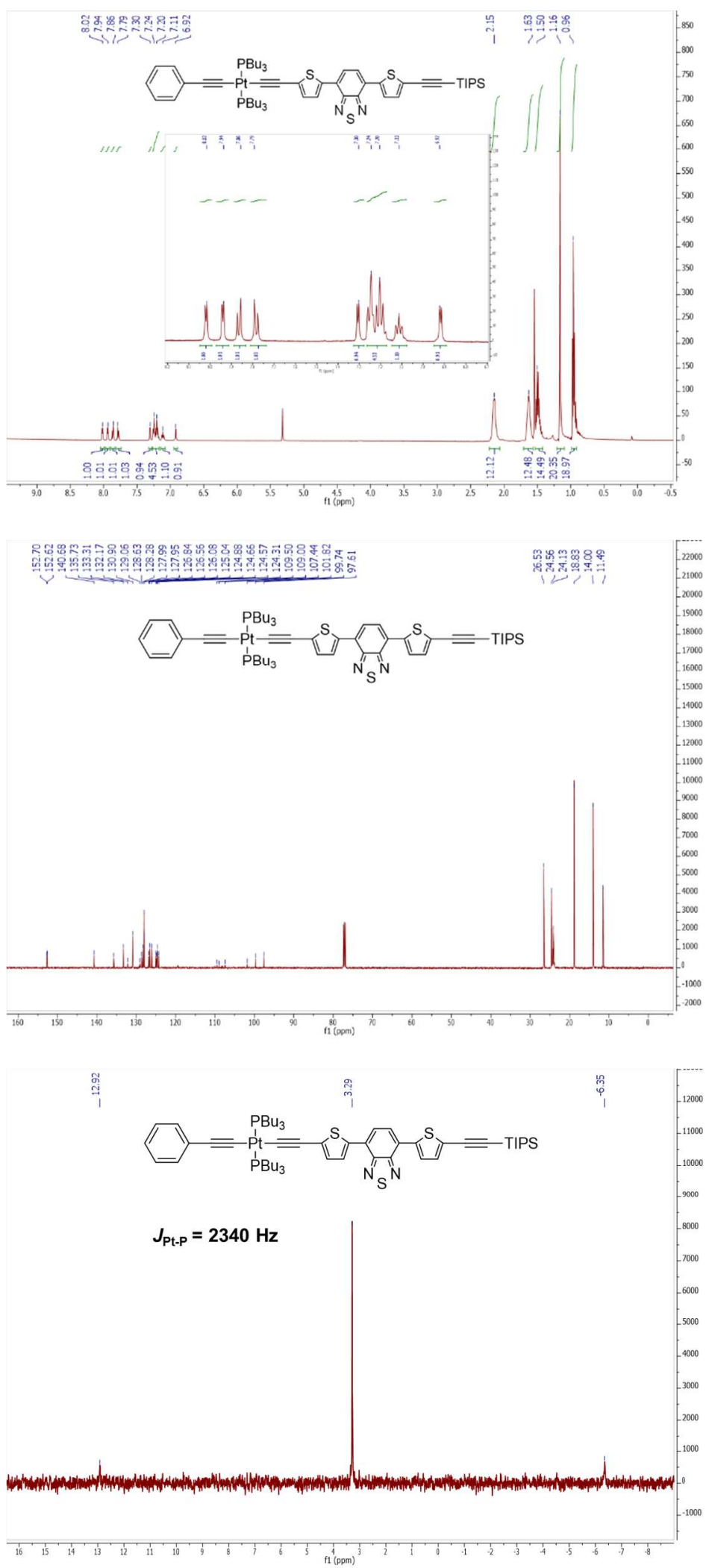

Figure S7. H, C and P-NMR of $\mathbf{1 0 .}$

Supporting Information. Cekli S., Winkel R. W., Schanze K. S. 


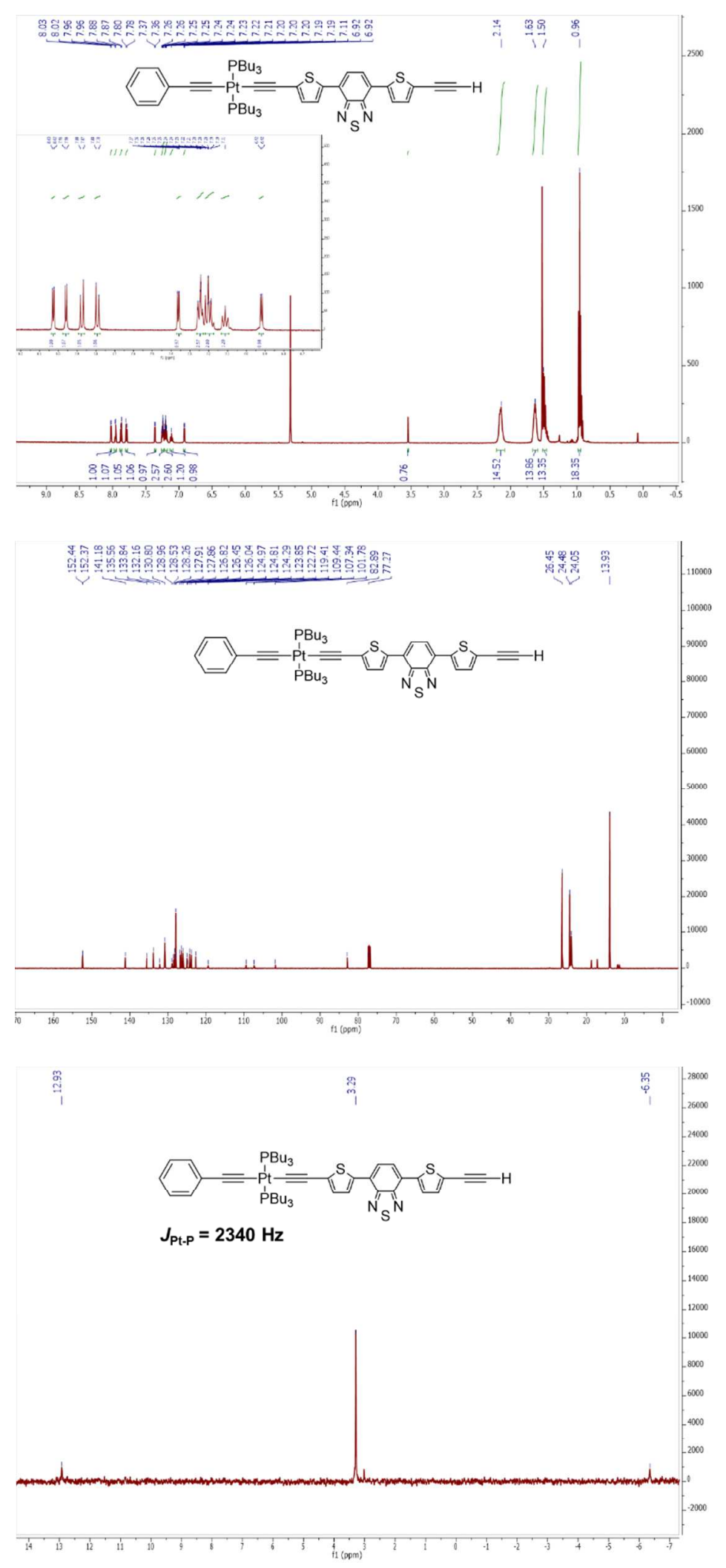

Figure S8. H, C and P-NMR of 11.

Supporting Information. Cekli S., Winkel R. W., Schanze K. S. 

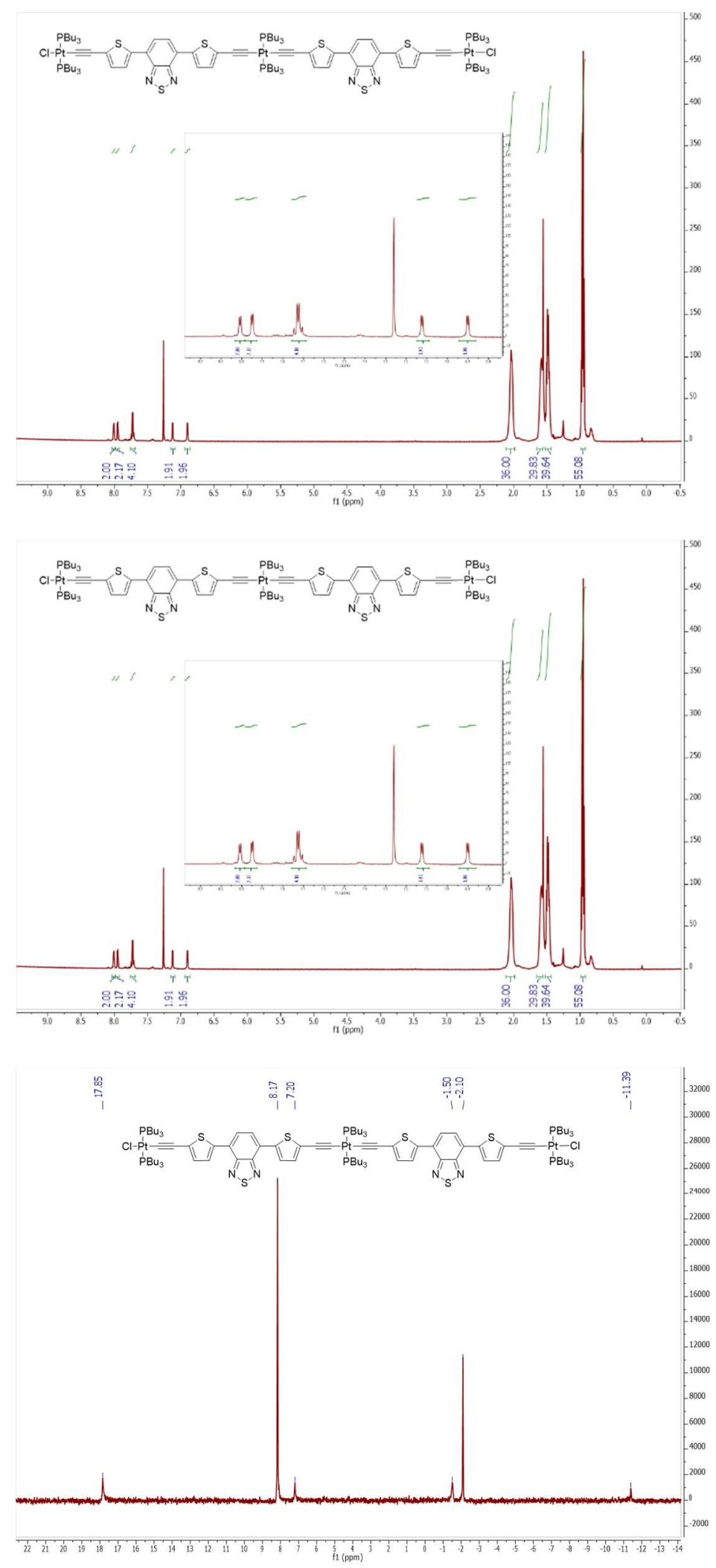

Figure S9. H, C and P-NMR of 12.

Supporting Information. Cekli S., Winkel R. W., Schanze K. S. 

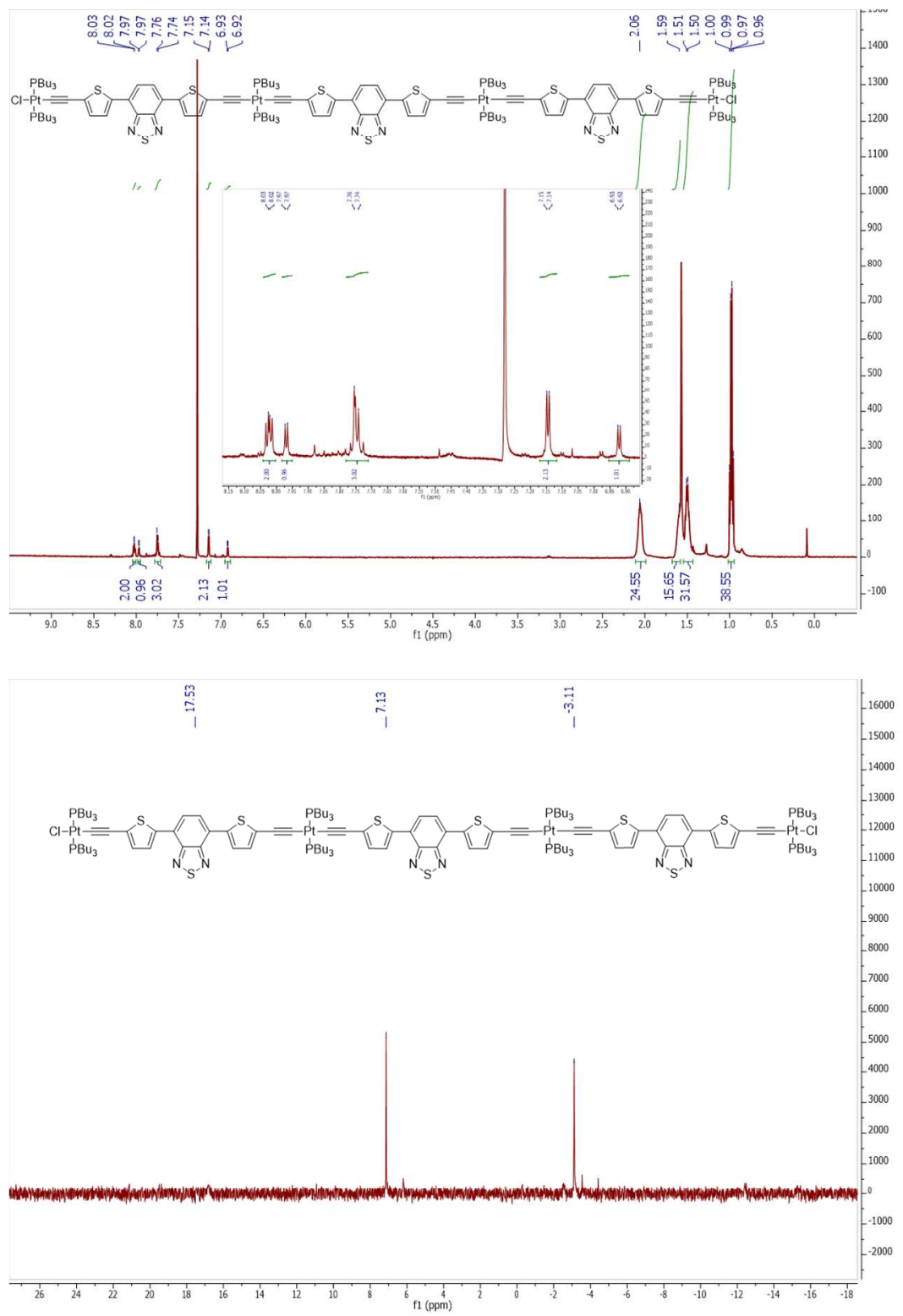

Figure S10. H, C and P-NMR of 13.

Supporting Information. Cekli S., Winkel R. W., Schanze K. S. 

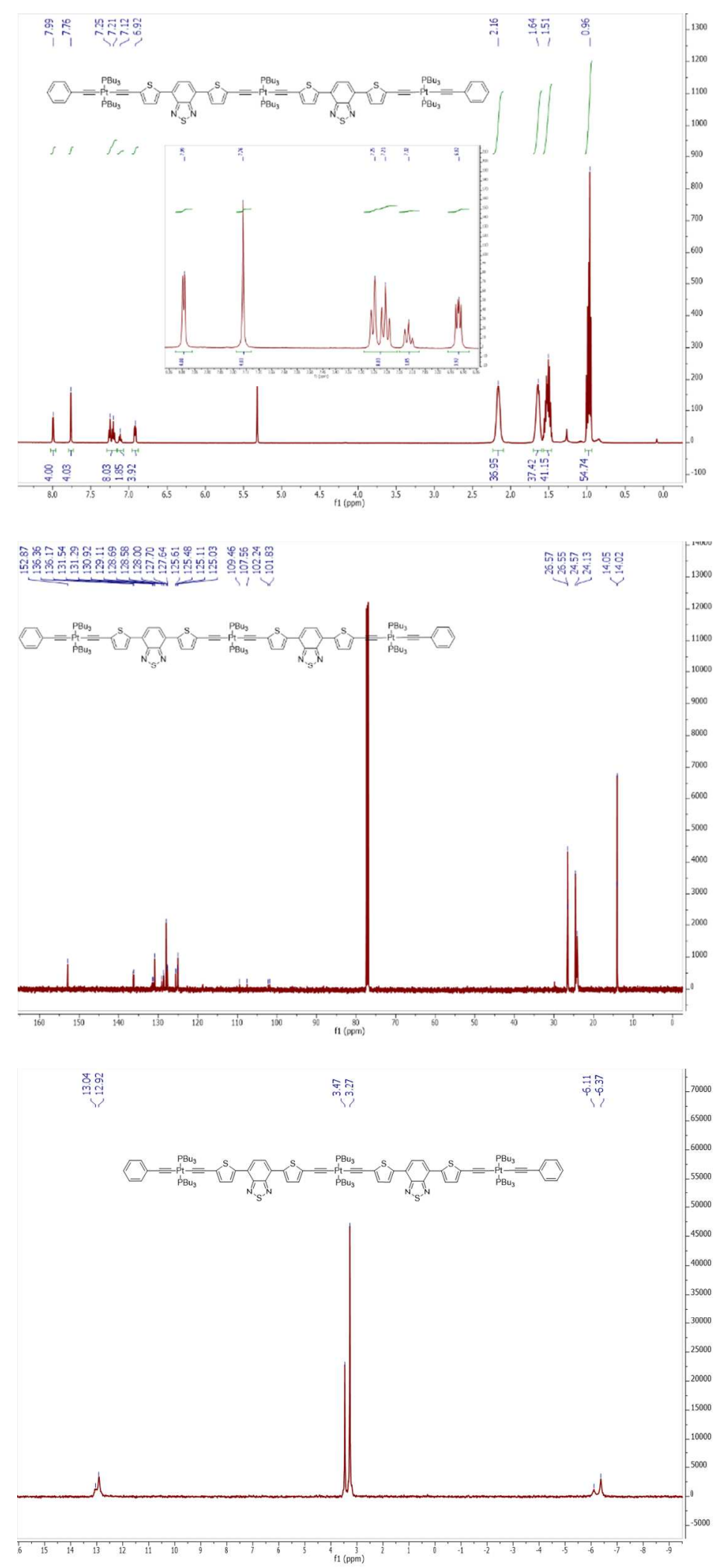

Figure S11. H, C and P-NMR of $\mathbf{O 2}$.

Supporting Information. Cekli S., Winkel R. W., Schanze K. S. 

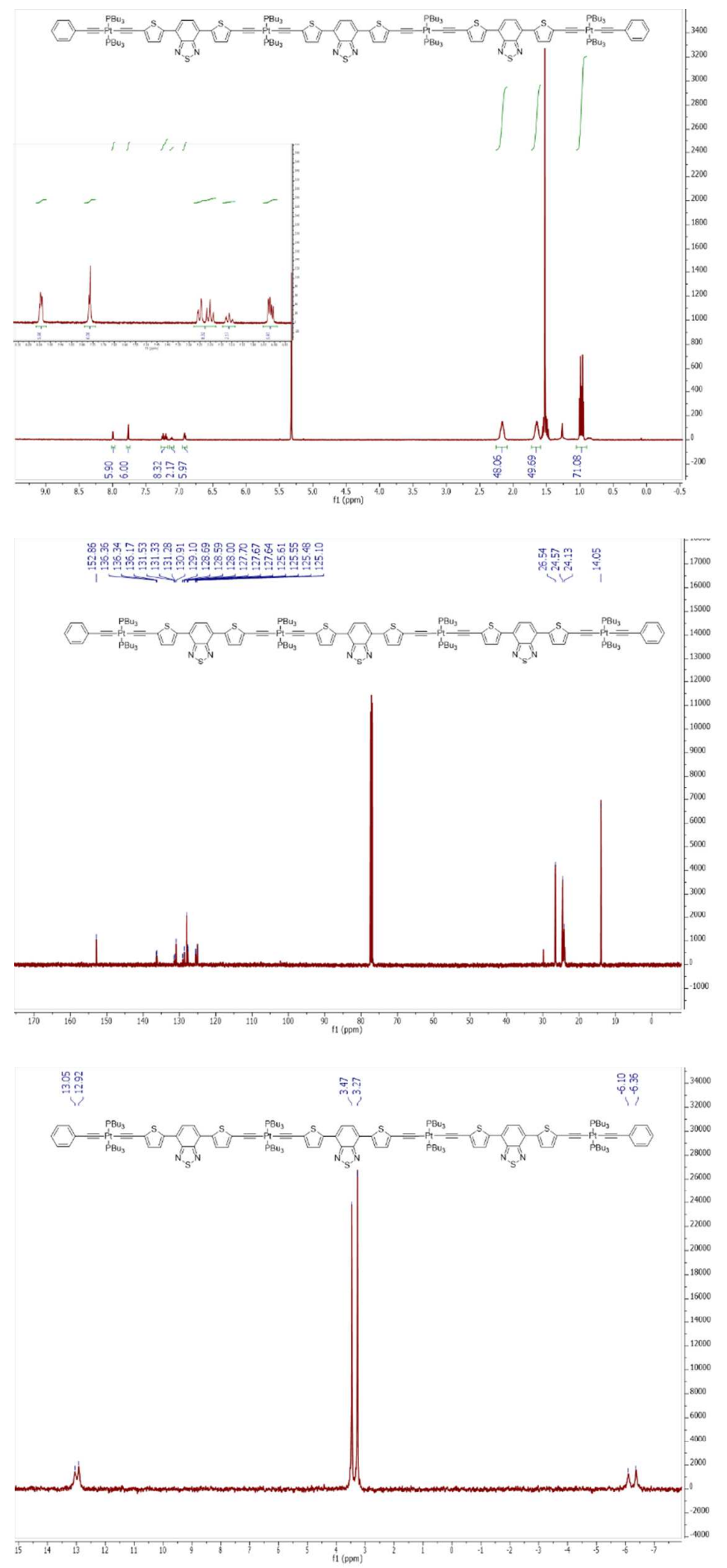

Figure S12. H, C and P-NMR of $\mathbf{O 3 .}$

Supporting Information. Cekli S., Winkel R. W., Schanze K. S. 

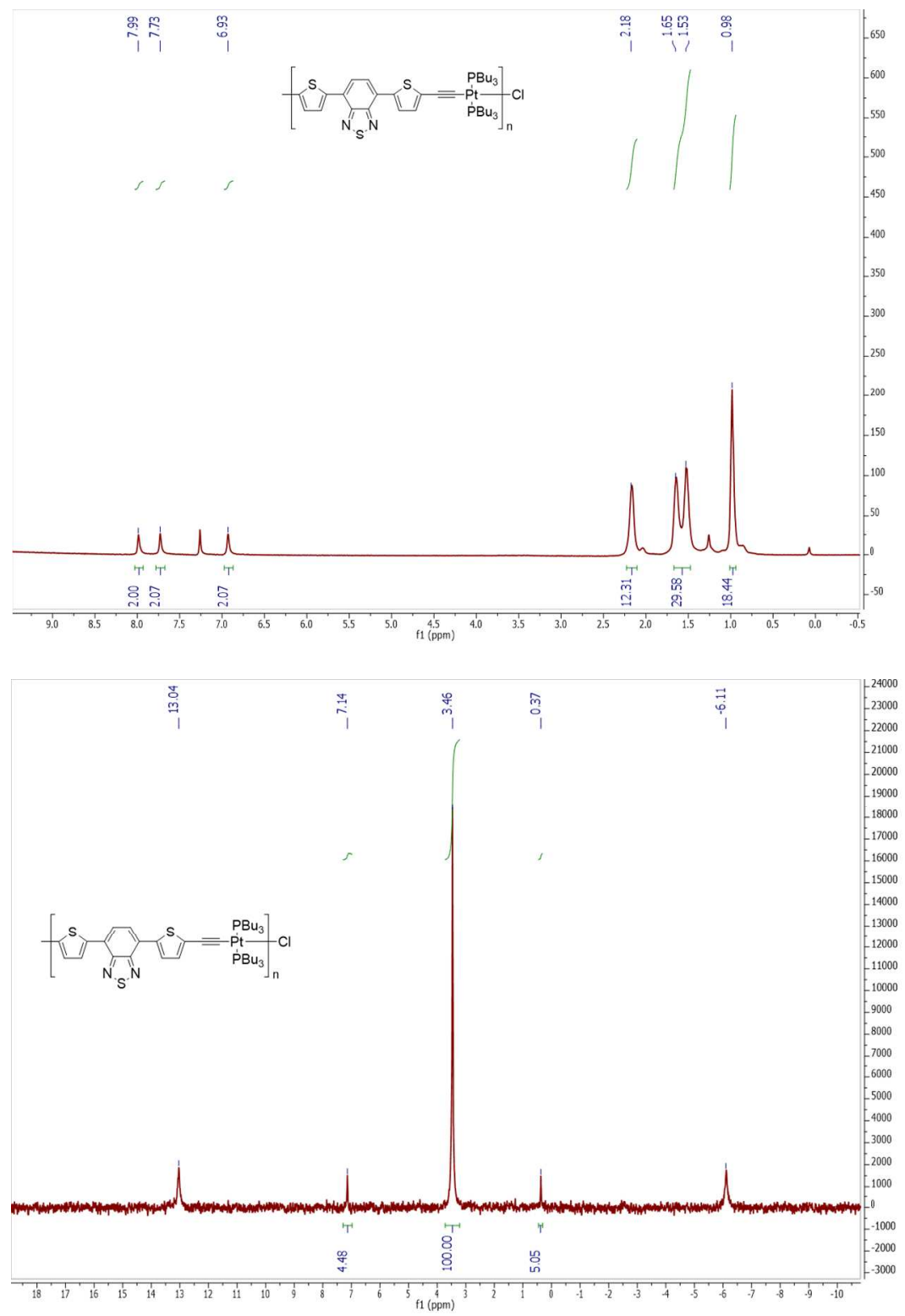

Figure S13. H and P-NMR of p-TBT.

Supporting Information. Cekli S., Winkel R. W., Schanze K. S. 


\section{Electrochemical Data}
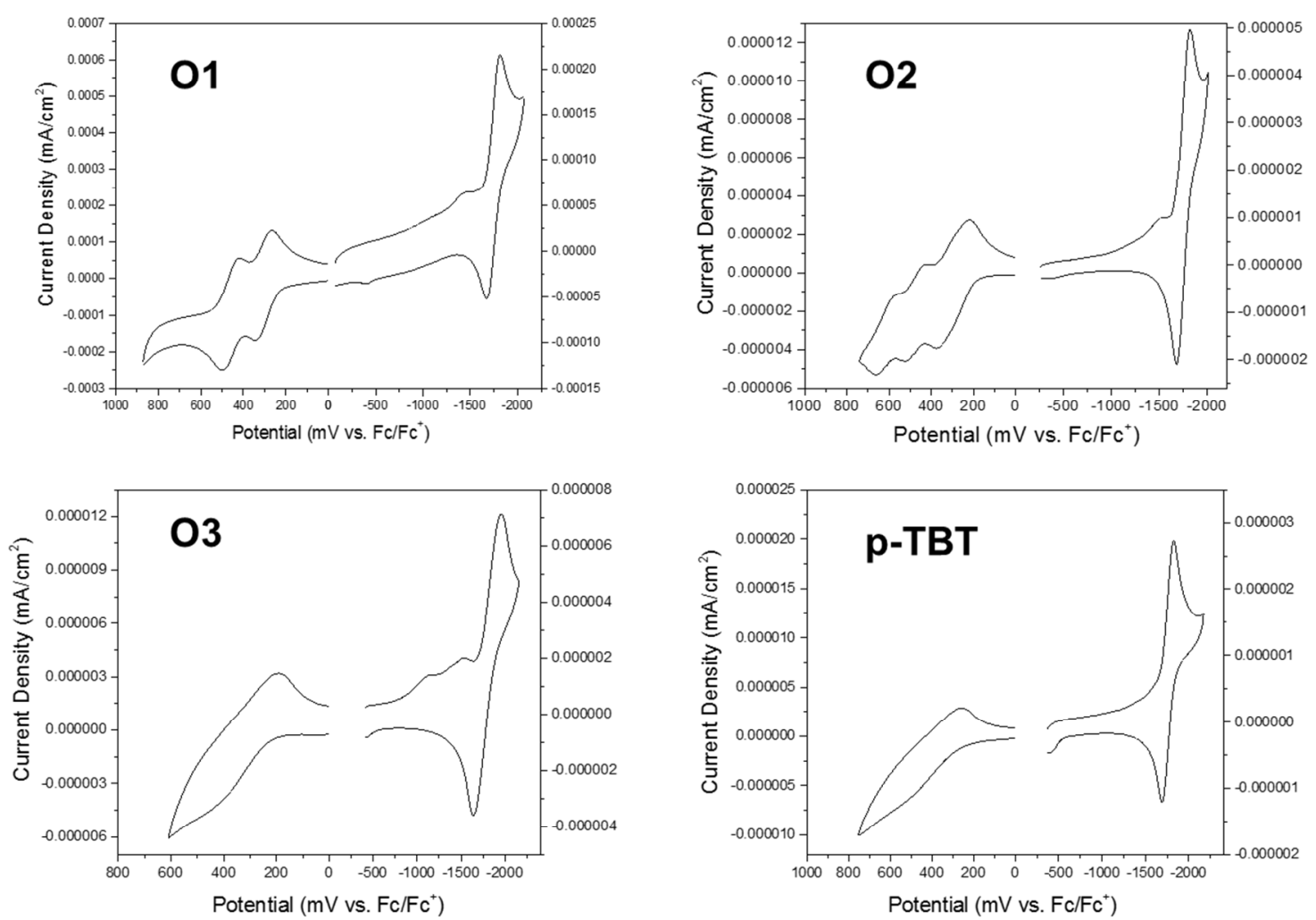

Figure S14. Cyclic voltammograms of O1-O3 and p-TBT.

Supporting Information. Cekli S., Winkel R. W., Schanze K. S. 


\section{Photophysical Data}
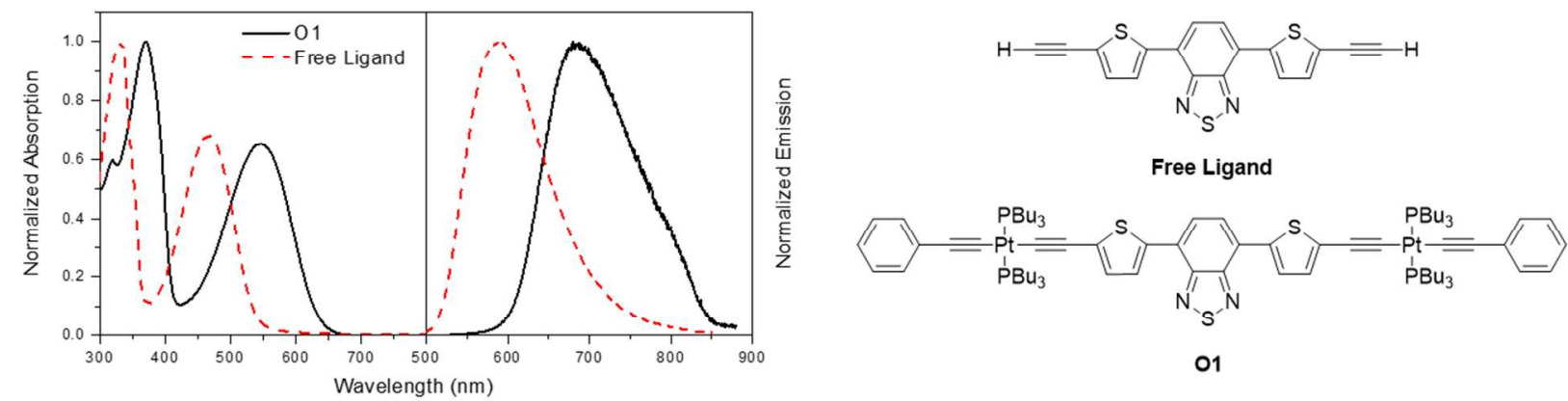

Figure S15. Normalized absorption and emission spectra of $\mathbf{O 1}$ and its free ligand.

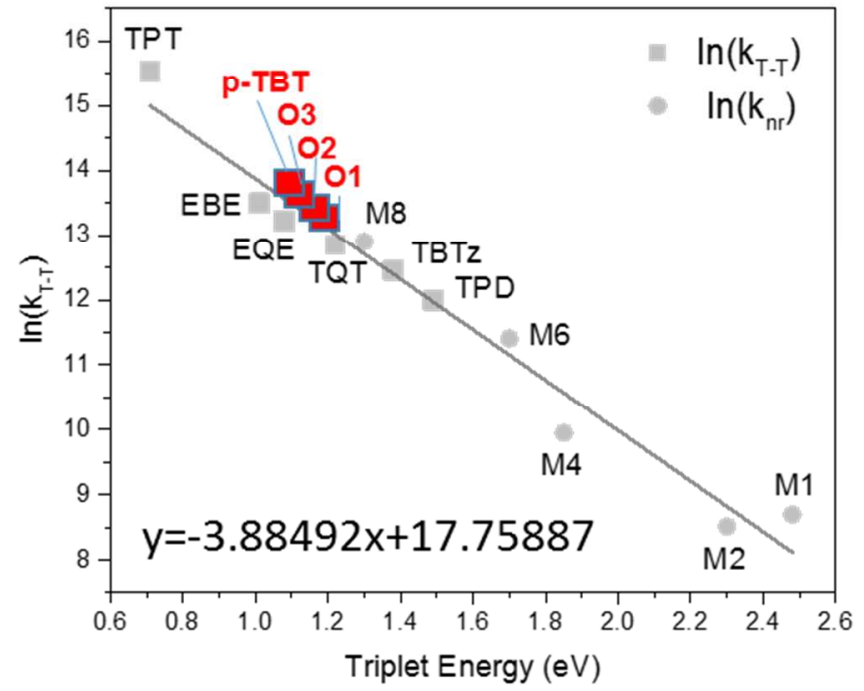

Figure S16. Energy gap law relationship. ${ }^{4}$

Supporting Information. Cekli S., Winkel R. W., Schanze K. S. 
a)

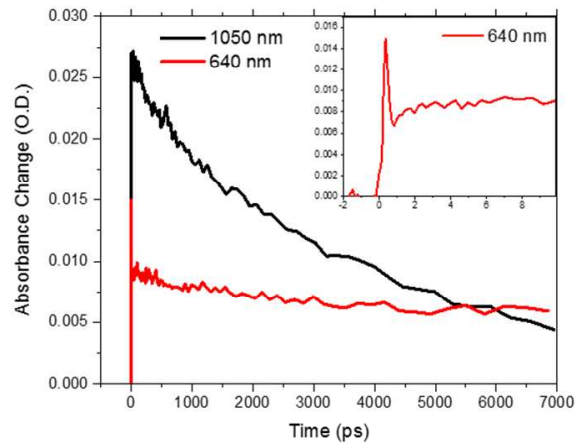

b)

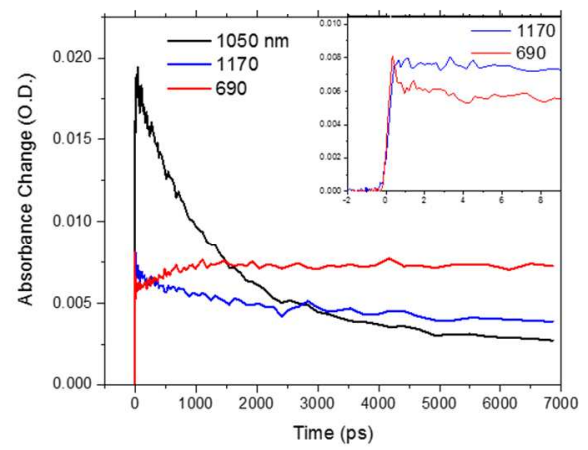

c)

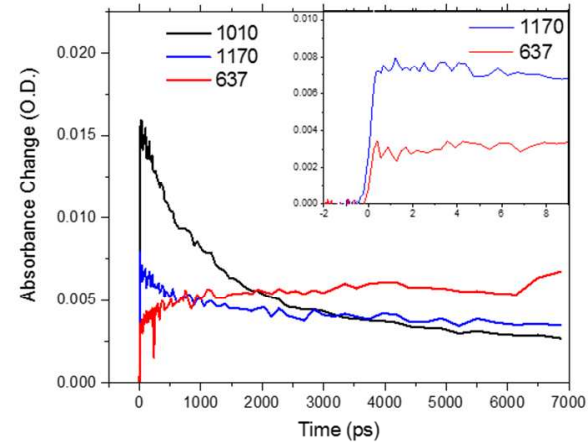

d)

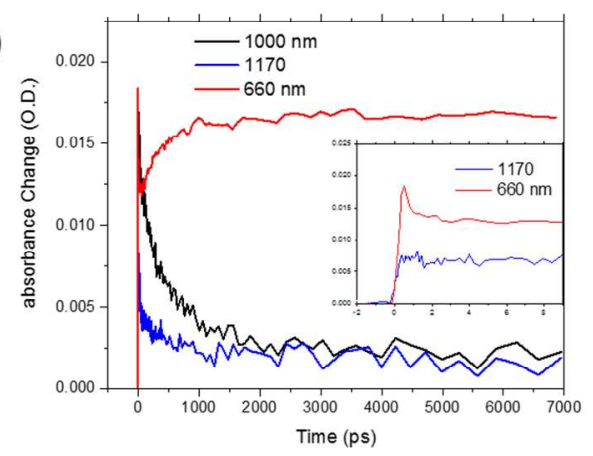

Figure S17. The temporal evolution of transient absorption at selected wavelengths. a) O1, b) O2, c) O3, d). p-TBT 


\section{Computational Data}

Table S1. Computed HOMO and LUMO energies of the lowest energy singlet conformation of the 01-03.

\begin{tabular}{ccc}
\hline & HOMO (eV) & LUMO (eV) \\
\hline O1 & -4.370 & -2.420 \\
O2 & -4.574 & -2.420 \\
O3 & -4.554 & -2.433 \\
\hline
\end{tabular}
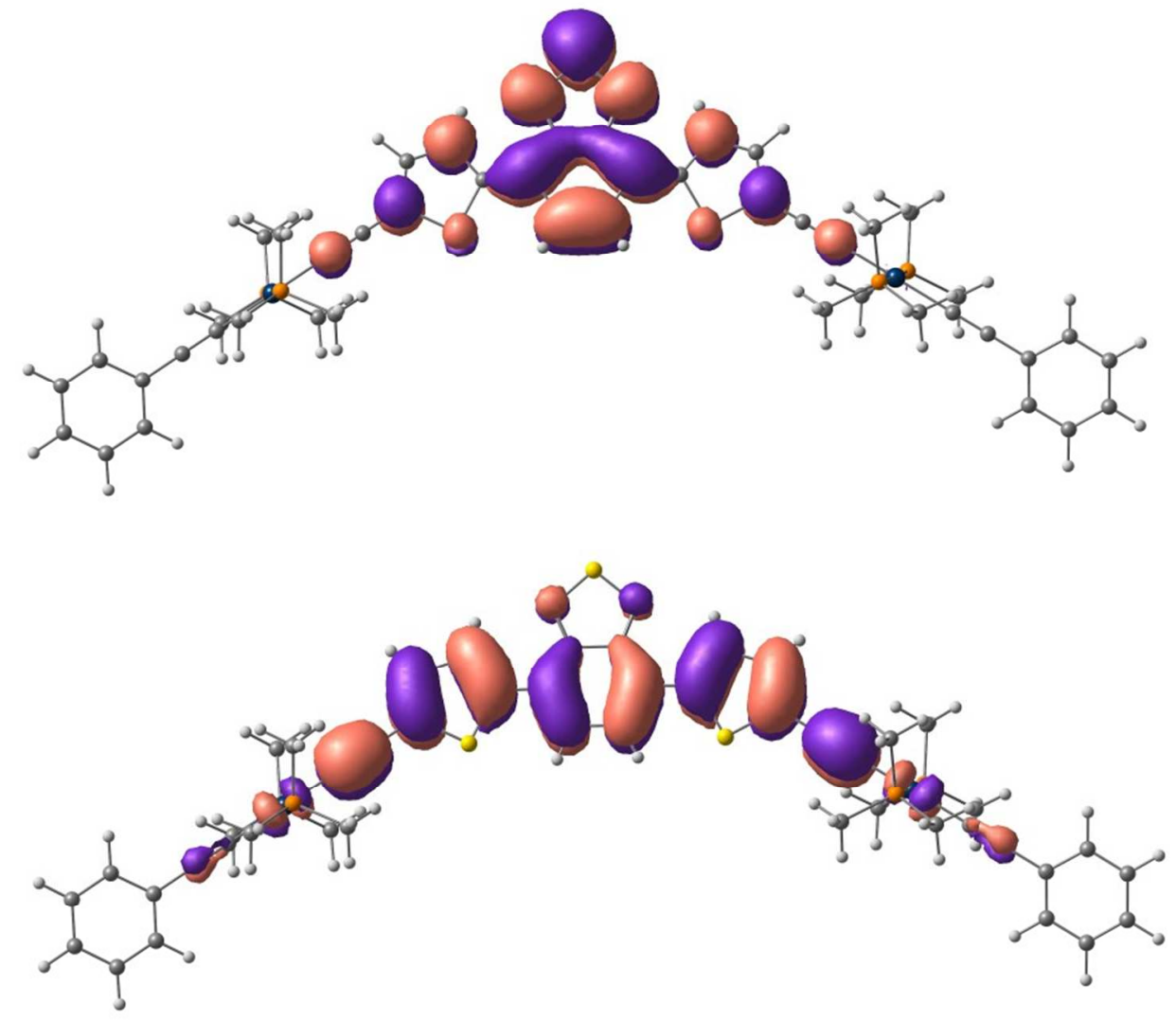

Figure S18. LUMO (top) and HOMO (bottom) of $\mathbf{O 1 .}$

Supporting Information. Cekli S., Winkel R. W., Schanze K. S. 

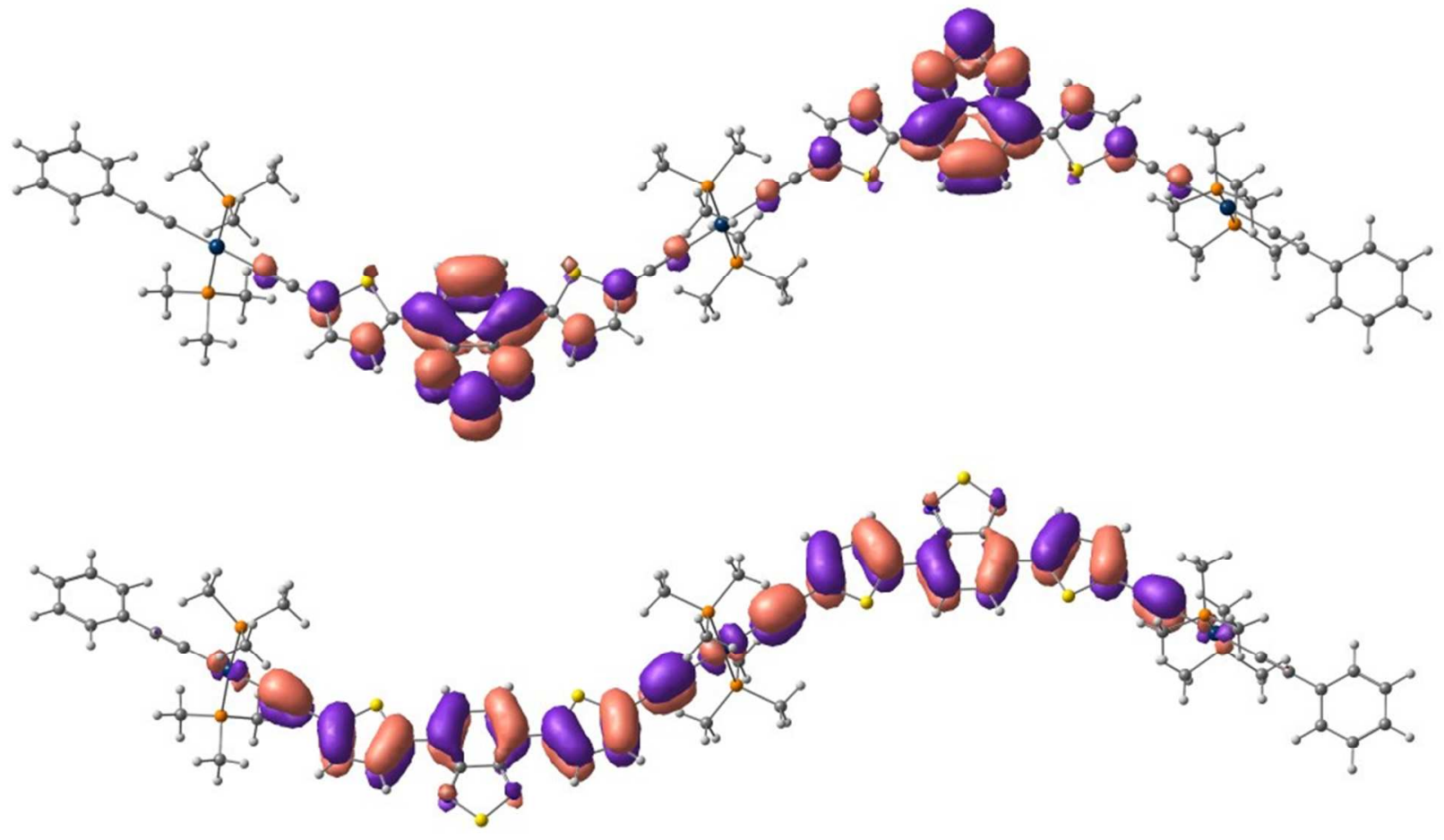

Figure S19. LUMO (top) and HOMO (bottom) of $\mathbf{O 2 .}$
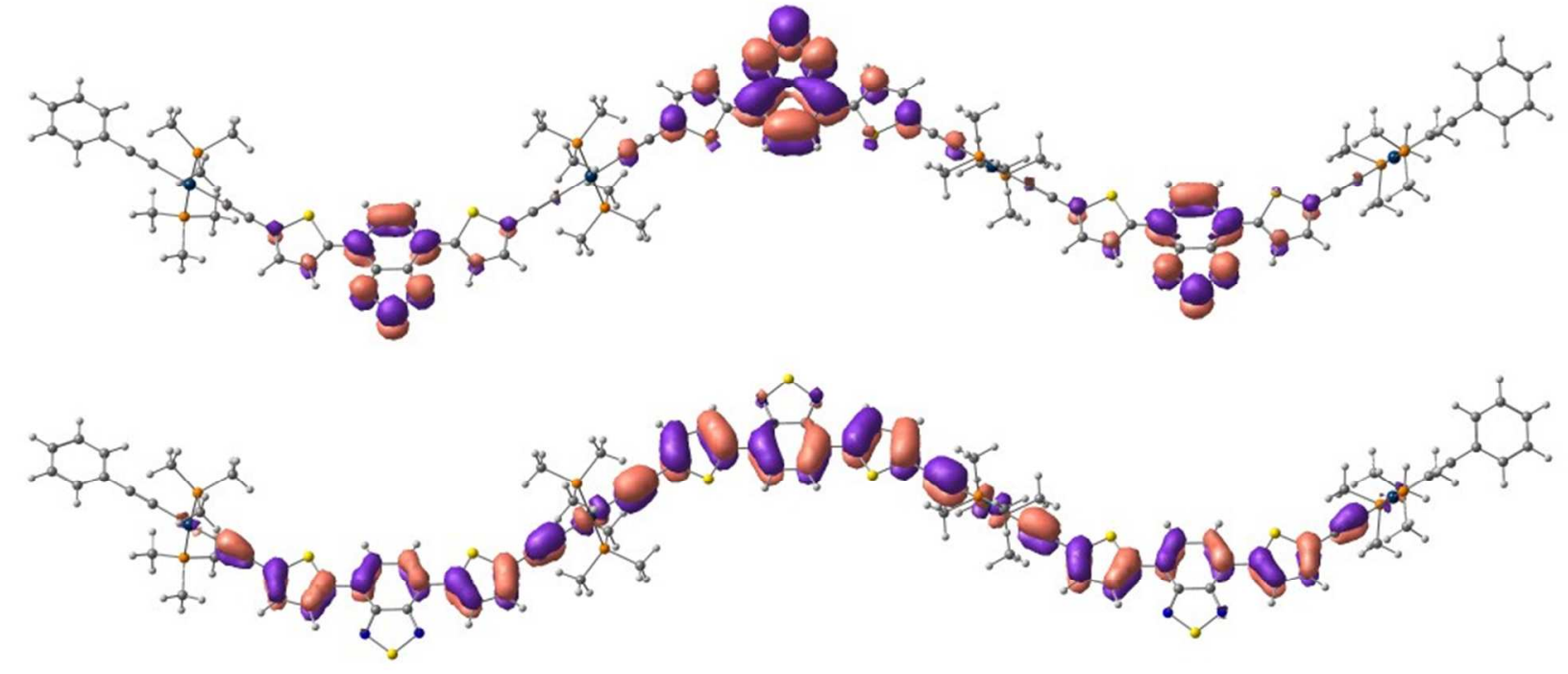

Figure S20. LUMO (top) and HOMO (bottom) of $\mathbf{O 3 .}$

Supporting Information. Cekli S., Winkel R. W., Schanze K. S. 


\section{References}

(1) Mei, J.; Ogawa, K.; Kim, Y. G.; Heston, N. C.; Arenas, D. J.; Nasrollahi, Z.; McCarley, T. D.; Tanner, D. B.; Reynolds, J. R.; Schanze, K. S. ACS Appl. Mater. Interfaces 2009, 1, 150-161.

(2) Mei, J., University of Florida, 2010.

(3) Chen, Z.; Grumstrup, E. M.; Gilligan, A. T.; Papanikolas, J. M.; Schanze, K. S. J. Phys. Chem. B 2013, 118, 372-378.

(4) Cekli, S.; Winkel, R. W.; Alarousu, E.; Mohammed, O. F.; Schanze, K. S. Chem. Sci. 2016. 